\title{
Stability and in vivo efficiency of natural cosmetic emulsion systems with the addition of vegetable oils
}

\author{
Jana Pavlačková ${ }^{*}$, Klára Kovacsová ${ }^{2}$, Petr Radiměřský ${ }^{2}$, Pavlína Egner ${ }^{1}$, Jana Sedlaříková1, \\ Pavel Mokrejš $\breve{3}^{3}$
}

\begin{abstract}
${ }^{1}$ Department of Fat, Surfactant and Cosmetics Technology, Faculty of Technology, Tomas Bata University in Zlin, Czech Republic, ${ }^{2}$ Laboratories of Natural Cosmetics, Nobilis Tilia, Krásná Lipa, Czech Republic, ${ }^{3}$ Department of Polymer Engineering, Faculty of Technology, Tomas Bata University in Zlin, Czech Republic
\end{abstract}

\begin{abstract}
The aim of the paper is to test stability and biophysical properties of hydrophilic and lipophilic emulsions with selected vegetable seed oils: Limnanthes alba, Prunus amygdalus dulcis, Cannabis sativa, Rosa rubiginosa and Hellianthus annuus. Biophysical properties of emulsions are investigated in vivo using non-invasive instrumental methods (corneometry, tewametry and $\mathrm{pH}$ ) in a group of 12 healthy women volunteers. Their stability profiles (colour, phase separation and centrifugation) under various temperatures $\left(9,25,37\right.$ and $57^{\circ} \mathrm{C}$ ) and storage time (24 hours, 2, 7, 14, 21 and 28 days) were monitored. The moisturising activities of the emulsions supplemented with various oils were comparable. The lipophilic emulsions showed a better ability to improve the condition of the skin barrier due to formation of a surface lipid film. The tested formulations regulated the $\mathrm{pH}$ of the skin towards neutral values. Lipophilic emulsions showed earlier phase separation and changes in colour. The greatest resistance to thermal stress during storage was observed for the emulsion bases. Emulsions containing oils, except for those with rosehip and hempseed oils, were stable up to the temperature of $37{ }^{\circ} \mathrm{C}$. The studied emulsion systems are excellent vehicles of vegetable oils and exhibit relatively good stability, benefiting the natural properties of skin.
\end{abstract}

Keywords: Vegetable oil/emulsion/stability. Skin/hydration. TEWL.

\section{INTRODUCTION}

Emulsions are some of the most widespread forms of cosmetic and pharmaceutical preparations. Emulsion is defined as a heterogeneous dispersion system of two immiscible liquids or liquids with limited miscibility, one of which being the dispersion part in the form of minute particles in the liquid environment of the other given liquid. In order to produce emulsions suitable for practical use, emulsifiers must be present. These are surface active agents that prevent degradation of the whole system (Imhof, Pine, 1997; Masmoudi et al., 2005). Two types of emulsions exist, based on polarity between the dispersion environments and dispersed substance, comprising oil in water $(\mathrm{o} / \mathrm{w})$ and water in oil $(\mathrm{w} / \mathrm{o})$.

\footnotetext{
*Correspondence: Jana Pavlačková. Department of Fat Surfactant and Cosmetics Technology, Faculty of Technology, Tomas Bata University in Zlín, Vavreckova 275, 76001 Zlín, Czech Republic. Tel.: +420 57603 1233. E-mail: pavlackova@ft.utb.cz
}

One of the most crucial properties of emulsions is stability, i.e. resistance to change in properties over time. Various methods for disrupting stability are available, for example, creaming and sedimentation are based on gravity separation. In both cases it is possible to observe gradual separation with the naked eye. Stability is a fundamental issue for the lifetime of an emulsion, or more precisely varied preparations, as any separation of components renders the given product unusable.

Application of emulsions both in cosmetics and in dermatological practice can be very diverse. Their use is determined by the specific properties of the active substances they have been incorporated with (Smaoui et al., 2012; Khan et al., 2010). Such substances include vegetable oils derived from various parts of plants. Indeed, oils represent one of the most common groups of materials utilized in the personal skin care products. Vegetable oils are defined as glycerol esters containing fatty acids called triglycerides, which constitute $95-98 \%$ of all oils. The remainder comprises unsaponifiable matter consisting of 
phospholipids, sterols, vitamins, and so on. One glycerol molecule containing two primary and one secondary alcohol group could be attached to one, two or even three fatty acids (Zielińska, 2014; Alander et al., 2006; Alvarez, Rodriguez, 2000). Varied representation of these acids in a glycerol molecule influences both the physical and sensorial properties of oils. The strongest cosmetic effect is usually found for unsaturated fatty acids, especially $\omega-3$ and $\omega-6$. In skin care, the most important oils are those with a high content of linoleic acid $(\omega-6)$ and $\alpha$-linoleic acid, as they are the least comedogenic and combat the occurrence of eczema. Oils can be applied directly to the skin or incorporated in a suitable carrier, such as an emulsion. The effect of oils is essentially that of greasing and protecting, thereby falling within the category of emollient. Although oils possess hydrophobic properties, hydration does not occur through the external supply of water, instead through the support of natural lipids in the stratum corneum, which ensures better functioning of the skin barrier. After applying the relevant oil, changes occur in the concentration profile of water in the stratum corneum (Stamatas et al., 2008). Cosmetic preparations primarily contain vegetable oils with an optimum proportion of fatty acids for the particular skin type. They create a protective film on the surface of the skin, which prevents evaporation of water and preserves the natural moisture and elasticity of the skin. Furthermore, they soften the skin, helping to reduce the appearance of inflammatory deposits and act as an antipruritic (Benatkova, 2010; Draelos, 2010; Fertekova, 2005; Kusmirek, 2005; Rele, Mohile, 2003; Miller, Miller, 1995).

Studies regarding hydration potential and stability of cosmetic emulsions containing seed oils and comparison of their properties on skin are somehow absent from the current literature. The aim of the paper is to test stability and biophysical properties of oil in water $(\mathrm{o} / \mathrm{w})$ and water in oil (w/o) cosmetic emulsions with the addition of selected vegetable seed oils: Limnanthes alba (meadowfoam), Prunus amygdalus dulcis (almond), Cannabis sativa (hemp), Rosa rubiginosa (roseship) and Hellianthus annuus (sunflower).

\section{MATERIAL AND METHODS}

\section{Vegetable seed oils}

Vegetable seed oils Limnanthes alba (meadowfoam), Prunus amygdalus dulcis (almond), Cannabis sativa (hemp), Rosa rubiginosa (roseship) and Hellianthus annuus (sunflower) were supplied by Nobilis Tilia (Czech Republic). Limnanthes alba oil contains more than $94 \%$ fatty acids mainly with a carbon chain length of C20 to C22, especially eicosenoic acid (Miwa, Wolff, 1962; Wolhman, 1997). Prunus amygdalus dulcis oil contains over $50 \%$ triacylglycerols, especially glycerides of oleic acid and linoleic acid (Miraliakbari, Shahidi, 2008; Orsavova et al., 2015). Cannabis sativa oil is a unique complex of polyunsaturated fatty acids, with the greatest presence of linoleic and $\alpha$-linoleic acid (Deferne, Pate, 1996; Leizer et al., 2000). In Rosa rubiginosa oil there are more than $77 \%$ of polyunsaturated fatty acids, above all linoleic and linolenic acids (Ilyasoglu, 2014). Hellianthus annuus oil is distinctive for high proportion of unsaturated fatty acids - linoleic and oleic (Rafalowski et al., 2008). Fatty acid composition of seed oils is summarized in Table I.

TABLE I - Fatty acids composition of seed oils

\begin{tabular}{lccccc}
\hline \multirow{2}{*}{ Fatty acid } & \multicolumn{5}{c}{ Content in oils (\%) } \\
\cline { 2 - 6 } & Meadowfoam & $\begin{array}{c}\text { Prunus amygdalus } \\
\text { dulcis }\end{array}$ & $\begin{array}{c}\text { Cannabis } \\
\text { sativa }\end{array}$ & $\begin{array}{c}\text { Rosa } \\
\text { rubiginosa }\end{array}$ & $\begin{array}{c}\text { Hellianthus } \\
\text { annuus }\end{array}$ \\
\hline Palmitic (C16:0) & - & $6-8$ & $5-7$ & $3-5$ & $4-9$ \\
Stearic (C18:0) & - & $0.5-2$ & $1-2$ & $1-2$ & $1-7$ \\
Oleic (C18:1) & - & $64-86$ & $8-13$ & $14-16$ & $14-40$ \\
Linoleic (C18:2) & - & $20-30$ & $50-60$ & $43-49$ & $48-74$ \\
Linolenic (C18:3) & - & 0.4 & $23-29$ & $32-38$ & 0.16 \\
Eicosenoic (C20:1) & $58-63$ & 0.16 & - & 0.45 & - \\
Eicosadienoic (C20:2) & 12 & - & - & 0.15 & - \\
Arachidonic (C20:4) & - & - & - & 2.1 & - \\
Erucic (C22:1) & 15 & - & - & - & - \\
Docosadienoic (C22:2) & $10-17$ & - & - & - & - \\
\hline
\end{tabular}




\section{Preparation of emulsion bases and formulations}

Two emulsion bases were used - hydrophilic (o/w) and lipophilic (w/o) supplied by Nobilis Tilia (Czech Republic) formulated entirely on a natural basis; their composition is provided in Tables II and III. Both types of emulsion lacked the presence of humectants and contained a dispersant in aqueous phase for simple incorporation of the oil. For both bases, the oil and aqueous phase were heated separately in a water bath of $60-70^{\circ} \mathrm{C}$. As regards the hydrophilic base, the aqueous phase was stirred until all of the xanthan gum was dissolved and the oil phase was added into the aqueous phase with constant stirring. For the lipophilic base, the aqueous phase was poured into the oil phase under constant stirring. Both emulsions were homogenized using a high-speed dispersing machine Ultra-Turrax TP18/2N (IKA Janke \& Kungel GmbH \& Co. KG, Germany) at 3,500 rpm. Afterwards, the mixture was allowed to cool to the temperature of $28^{\circ} \mathrm{C}$. Vegetable seed oils at the amount of $3 \%(\mathrm{w} / \mathrm{w})$ were homogenized into the emulsion bases on a RZR 2020 stirrer (Heidolph, Germany) at 2,000 rpm for 10 minutes at $24{ }^{\circ} \mathrm{C}$. The $\mathrm{pH}$ of the prepared formulations was as follows: 4.38 in the hydrophilic emulsion base, 4.41-4.49 in hydrophilic emulsions with vegetable oils; 6.13 in the lipophilic base, 6.19-6.24 in lipophilic emulsions with vegetable oils.

\section{Stability and centrifugation test}

$6.0 \mathrm{~g}$ samples for tests on stability and centrifugation were collected immediately after preparing the emulsion formulations containing vegetable oils in centrifugal tubes. These samples were maintained in an incubator at the following temperatures: $9.0 \pm 0.1{ }^{\circ} \mathrm{C}, 25.0 \pm 0.1{ }^{\circ} \mathrm{C}$, $37.0 \pm 0.1{ }^{\circ} \mathrm{C}$, and $57.0 \pm 0.1{ }^{\circ} \mathrm{C}$ at $75.0 \pm 3.0 \%$ relative humidity. The properties of the emulsions were observed at intervals of 24 hours after preparation, and then after 2, 7, 14,21 and 28 days. Stability tests, including centrifugation, were also carried out immediately after the samples had been prepared, and then repeatedly at the same intervals as the previous stability tests. Centrifugation was carried out at 5,000 rpm and $24{ }^{\circ} \mathrm{C}$ for $10 \mathrm{~min}$.

\section{Volunteers}

Measurements were performed on 12 healthy women (aged 19 to 49 years, mean age 32 years) with no history of atopic eczema or other skin diseases. This group of volunteers was measured for the effectiveness of both emulsion systems $-\mathrm{o} / \mathrm{w}$ and $\mathrm{w} / \mathrm{o}$ with the addition of vegetable oils. The method of selecting the volunteers
TABLE II - Composition of hydrophilic base of emulsion

\begin{tabular}{ll}
\hline INCI name & Function of ingredient \\
\hline \multicolumn{1}{c}{ Oil phase } \\
\hline $\begin{array}{l}\text { Caprylic/Capric Triglyceride } \\
\text { Alcohol, Sucrose Stearate, }\end{array}$ & Neutral oil/Filler \\
Sucrose Tristearate & Primary emulsifiers \\
Cetyl Alcohol & Stabilizer/co-emulsifier/ \\
& thickening agent \\
Stearin & Stabilizer/co-emulsifier/ \\
& thickening agent \\
Tocopherol Acetate & Antioxidant \\
\hline \multicolumn{2}{c}{ Aqueous phase } \\
\hline Xantham Gum & Thickening agent/stabilizer \\
Polyglyceryl-5 Oleate & Secondary emulsifier \\
Sodium Benzoate, Potassium & Preservative \\
Sorbate, Aqua & \\
Citric Acid & pH regulator \\
Aqua & Solvent \\
\hline
\end{tabular}

TABLE III - Composition of lipophilic base of emulsion

\begin{tabular}{|c|c|}
\hline INCI name & Function of ingredient \\
\hline \multicolumn{2}{|c|}{ Oil phase } \\
\hline Caprylic/Capric Triglyceride & Filler \\
\hline $\begin{array}{l}\text { Polyglyceryl-4 Diisostearate/ } \\
\text { Polyhydroxystearate/Sebacate }\end{array}$ & Primary emulsifier \\
\hline Hydrogenated Castor Oil & $\begin{array}{l}\text { Stabilizer/co-emulsifier/ } \\
\text { thickening agent }\end{array}$ \\
\hline Cera Alba & $\begin{array}{l}\text { Stabilizer/co-emulsifier/ } \\
\text { thickening agent }\end{array}$ \\
\hline Magnesium Stearate & Thickening agent \\
\hline \multicolumn{2}{|c|}{ Aqueous phase } \\
\hline Magnesium Sulphate & Stabilizer \\
\hline $\begin{array}{l}\text { Sodium Benzoate, Potassium } \\
\text { Sorbate, Aqua }\end{array}$ & Preservative \\
\hline Aqua & Solvent \\
\hline
\end{tabular}

and the testing itself were conducted in accordance with International Ethical Guidelines for Health-related Research Involving Humans prepared by the Council for International Organizations of Medical Sciences (CIOMS, 2016). All volunteers gave their informed consent prior to inclusion in the study. For 12 hours prior to and during the study the volunteers were not allowed to apply any topical cosmetic products, only evening shower water was permitted. 


\section{Instrumental techniques}

Non-invasive methods are used in the field of experimental dermatology and cosmetology, enabling quantitative evaluation of parameters which describe the barrier function of the skin. These methods were also applied in this study. The water content in stratum corneum was measured with a corneometrical probe - the Corneometer ${ }^{\circledR}$ CM 825 (Courage \& Kazaka Electronic, Cologne, Germany). Its function is based on evaluating changes in electric capacity on the surface of the skin while utilizing the relatively high dielectric constant of water. The results are displayed in arbitrary units (a. u.). Another parameter for the survey was transepidermal water loss (TEWL), which was monitored with a probe the Tewameter ${ }^{\circledR}$ TM 300 (Courage \& Kazaka Electronic, Cologne, Germany). In general, the value to be determined is the flow of water vapour over the stratum corneum into the space of an open chamber that is cylindrical in shape, featuring two pairs of sensors for temperature and the relative humidity of air. TEWL is calculated from the difference between the two measurement points using Fick's law of diffusion and displayed in grams per hour per square meter $\left(\mathrm{g} \cdot \mathrm{m}^{-2} \mathrm{~h}^{-1}\right)$. In order to determine the acidity of the acid mantle, the PH 905 skin-pH-meter ${ }^{\circledR}$ (Courage \& Kazaka Electronic, Cologne, Germany) was utilized. Its specially designed probe consists of a flat-topped glass electrode for full skin contact, connected to a voltmeter. The system measures potential changes due to the activity of hydrogen cations surrounding the very thin layer of semisolid forms at the top of the probe. The changes in voltage are displayed in terms of $\mathrm{pH}$.

\section{Study design of biophysical measurements}

Measurements were carried out in an air-conditioned room (temperature $22-24{ }^{\circ} \mathrm{C}$, relative humidity $45-50 \%$ ). All measurements were performed after a rest of $20 \mathrm{~min}$ for equilibration. The volar sides of both the left and right forearm were divided into 5 test areas, each measuring 8 $\mathrm{cm}^{2}$. Concurrently, untreated skin remained on the volar side of the left forearm of each volunteer, which was kept in order to provide comparison in case of any irritative reactions incurred on the skin. Other areas on the volar forearm were pre-treated with a $0.5 \%(\mathrm{w} / \mathrm{w})$ solution of sodium lauryl sulphate (SLS), (Sigma-Aldrich, Czech Republic) in saline for 4 hours. After irritation, each site tested was measured for hydration via the corneometric probe, while for TEWL the tewameter was applied, and the $\mathrm{pH}$ probe tested for acidity of the skin surface. The emulsion base and base with homogenized oils were gradually applied on each site. The effects of the applied formulations on the stratum corneum were monitored in all volunteers after 1, 2, 3, 4, 24 and 48 hours, in the same order as after treatment with SLS. Hydration was measured at each test site at least five times. TEWL measurement was carried out fifteen times at each test site. Since this is dependent on the temperature of the epidermis, the environment and the probe itself, the first five values from such measurement were eliminated.

\section{Processing the measured parameters}

The physical properties of the emulsions, i.e. phase separation and colour during stability and centrifugation tests, were assessed visually. Separation of phases was evaluated according to the following scale: release of the added oil component onto the surface of the emulsion $(+)$; visible separation of phases $(++)$; continuing separation of phases $(+++)$; change in colour of emulsion $(+)$. The biophysical properties obtained after applying the emulsions to the skin were processed using descriptive statistics; mean and standard deviation (SD) under Microsoft Excel Professional Plus 10 were calculated.

\section{RESULTS AND DISCUSSION}

\section{Stability of formulated emulsions}

Most cosmetics and pharmaceutical formulations are oil in water $(\mathrm{o} / \mathrm{w})$ or water in oil (w/o) emulsions. From a physical perspective, these are thermodynamically unstable systems whose instability is expressed by division into two distinct phases (Masmoudi et al., 2005). Consequently, the formulations tested herein were stored under various conditions. Changes in phase separation and colour are shown in Table IV. The findings that failed to reveal any changes in the emulsion samples, i.e. results from storing samples at temperatures of $9{ }^{\circ} \mathrm{C}$ and $25^{\circ} \mathrm{C}$ for 24 hours and two days, are not displayed here. The freshly prepared emulsions were white in shade. The first small changes in colour towards creamy-white to yellow were observed in hydrophilic emulsion after 21 days of storage at $57{ }^{\circ} \mathrm{C}$ and in lipophilic emulsion after only 28 days of storage at the same temperature. Alteration in colour at the end of the experiment was probably caused by separation of the oil phase of the emulsion, which may occur at higher temperatures.

The results of tests carried out with the hydrophilic emulsion base and emulsion formulations containing meadowfoam (Limnanthes alba), almond (Prunus amygdalus dulcis) and sunflower (Hellianthus annuus) 
Stability and in vivo efficiency of natural cosmetic emulsion systems with the addition of vegetable oils

TABLE IV - Physical characteristics of vehicle and emulsions with oils kept at $37^{\circ} \mathrm{C}$ and $57^{\circ} \mathrm{C}$

\begin{tabular}{|c|c|c|c|c|c|c|c|c|c|c|c|c|c|c|c|c|c|}
\hline \multirow{3}{*}{$\begin{array}{l}\text { Emulsion } \\
\text { Parameter } \\
\text { Test } \\
\text { Days } \\
\end{array}$} & & \multicolumn{8}{|c|}{ Hydrophilic } & \multicolumn{8}{|c|}{ Lipophilic } \\
\hline & & \multicolumn{4}{|c|}{ Phase separation } & \multicolumn{4}{|c|}{ Colour } & \multicolumn{4}{|c|}{ Phase separation } & \multicolumn{4}{|c|}{ Colour } \\
\hline & & \multicolumn{8}{|c|}{ Stability/Centrifugation } & \multicolumn{8}{|c|}{ Stability/Centrifugation } \\
\hline Emulsion base (EB) & & $-1-$ & $-1-$ & $-1-$ & $-/+$ & $-1-$ & $-1-$ & $-1-$ & $-1-$ & $-1-$ & $-1-$ & $-1-$ & $-1+$ & $-1-$ & $-1-$ & $-1-$ & $-1-$ \\
\hline EB+Meadowfoam & & $-1-$ & $-1-$ & $-1-$ & $-1+$ & $-1-$ & $-1-$ & $-1-$ & $-1-$ & $-1-$ & $-1-$ & $-1-$ & $-1+$ & $-1-$ & $-1-$ & $-1-$ & $-1-$ \\
\hline $\mathrm{EB}+\mathrm{Cannabis}$ sativa & $\hat{n}$ & $-1-$ & $+/+$ & $+/+$ & $+/+$ & $-1-$ & $-1-$ & $-1-$ & $-1-$ & $-1-$ & $-1-$ & $-1+$ & $-/++$ & $-1-$ & $-1-$ & $-1-$ & $-1-$ \\
\hline EB+Rosa rubiginosa & & $-1-$ & $-1-$ & $+/+$ & $+/+$ & $-1-$ & $-1-$ & $-1-$ & $-1-$ & $-1-$ & $-1-$ & $-1+$ & $-1+$ & $-1-$ & $-1-$ & $-1-$ & $-1-$ \\
\hline EB+Hellianthus annuus & & $-1-$ & $-1-$ & $-1-$ & $-1+$ & $-1-$ & $-1-$ & $-1-$ & $-1-$ & $-1-$ & $-1-$ & $-1-$ & $-1+$ & $-1-$ & $-1-$ & $-1-$ & $-1-$ \\
\hline Emulsion base (EB) & & $-1-$ & $-1-$ & $-1+$ & $+/+++$ & $-1-$ & $-1-$ & $-1+$ & $+/+$ & $-1-$ & $-1+$ & $+/+$ & $+/+$ & $-1-$ & $-1-$ & $-1-$ & $-1+$ \\
\hline EB+Meadowfoam & & $-1-$ & $-1+$ & $-/++$ & +/+++ & $-1-$ & $-1-$ & $-/+$ & $+/+$ & $-1-$ & $+/-$ & $+/+$ & $++/+$ & $-1-$ & $-1-$ & $-1-$ & $-/+$ \\
\hline EB+Hellianthus annuus & & $-1-$ & $-/+$ & $-/++$ & $++/+++$ & $-1-$ & $-/+$ & $-/+$ & $+/+$ & $-1-$ & $+/-$ & $+/+$ & $+/++$ & $-1-$ & $-1-$ & $-1-$ & $+/+$ \\
\hline
\end{tabular}

Note: added oil component released on the surface of the emulsion $(+)$; visible phase separation $(++)$; continuing phase separation $(+++)$; change in colour of emulsion $(+)$

oils showed their stability under all storage conditions at $9,25,37$ and $57^{\circ} \mathrm{C}$ for the period of 21 days. At higher temperatures, $37^{\circ} \mathrm{C}$ and $57^{\circ} \mathrm{C}$, phase separation was observed in hydrophilic emulsions supplemented with hemp (Cannabis sativa) oil after 14 days of testing and in rosehip oil after 21 days of storage. This instability was observed in the separation of an oil layer and, after 28 days of testing, phase separation had already become discernible. The presence of polyunsaturated fatty acids in these oils, especially $\alpha$-linolenic, limits their stability (Prescha et al., 2014).

The lipophilic base and formulations with various oils did not exhibit any alteration in phase separation or discolouration for 48 hours. In fact, the first physical changes were observed after 7 days of storage at $57^{\circ} \mathrm{C}$. At this point an expansion of frothy air bubbles occurred, which was probably worked into the emulsion during preparation. In the subsequent maintenance stage, the oil phase was released from the emulsions. The most stable of all proved to be the formulations containing almond (Prunus amygdalus dulcis) and sunflower (Hellianthus annuus) oils.

Stability in the behaviour of the emulsions was also observed after a repeated centrifugation test. This procedure uses centrifugal force to separate two immiscible liquids, which represents a useful tool for evaluating and predicting the lifetime of emulsions (Khan et al., 2010). No changes were observed in the hydrophilic base and emulsion formulation with almond (Prunus amygdalus dulcis) oil after centrifugation and storage at $9,25,37$ and $57^{\circ} \mathrm{C}$ until the $14^{\text {th }}$ day of storage. The greatest physical alterations were observed in hydrophilic formulations after 21 days of storage at $57^{\circ} \mathrm{C}$. After conducting repeated centrifugation tests of lipophilic emulsions, no phase separation occurred in the base or the emulsions with meadowfoam (Limnanthes alba), almond (Prunus amygdalus dulcis) and sunflower (Hellianthus annuus) oils until the $14^{\text {th }}$ day of storage. Destabilization was observed after the $21^{\text {st }}$ day at the temperature of 57 ${ }^{\circ} \mathrm{C}$. One week later, changes in separation were already being accompanied by alteration in colour in the lipophilic emulsions. Reduced resistance to centrifugation was detected in emulsions incorporated with hemp (Cannabis sativa) and rosehip (Rosa rubiginosa) oils.

During the experiment, it was possible to observe the disintegration of two different emulsion systems hydrophilic and lipophilic. Evaluating the stability tests clearly shows that instability in the lipophilic emulsions due to temperature was exhibited about one week earlier than in the case of hydrophilic emulsions. However, greater phase separation and changes in colour were observed more frequently in the hydrophilic formulations. The highest resistance to thermal stress was discovered only in the emulsion bases. In terms of individual observations made, it can be stated that vegetable oils, namely meadowfoam (Limnanthes alba), almond 
(Prunus amygdalus dulcis) and sunflower (Hellianthus annuus) oils, did not exert a great impact on the stability of emulsions. In contrast, hemp (Cannabis sativa) and rosehip (Rosa rubiginosa) oils actually decreased the stability of the emulsions. Nevertheless, most of the tested emulsion formulations supplemented with vegetable oils were stable up to the temperature of $37^{\circ} \mathrm{C}$. This storage temperature is necessary in order to maintain the unique composition of fatty acids and the stability of the emulsions. Both the emulsion systems studied could be described as suitable carriers of vegetable oils, exhibiting relatively good stability.

\section{Biophysical characteristics}

The biophysical effects of the given hydrophilic and lipophilic emulsions on skin, including formulations containing vegetable oils, are shown in Figures 1-3. The sites defined for application of the prepared formulations were pre-treated with $0.5 \%(\mathrm{w} / \mathrm{w})$ SLS solution in order to set initial conditions corresponding to the skin after a shower or washing with cosmetic preparations. This method of pre-treating the skin with SLS solution in various concentrations is referred to in a number of works that tested various preparations (Polaskova, Pavlackova, Egner, 2015; Lodén, Wessman, 2001; De Paepe et al., 2000; Lodén, Andersson, 1996; Lévêque et al., 1993).

Figures $1 \mathrm{a}$ and $\mathrm{b}$ show a noticeable decline in hydration of skin immediately upon application of the emulsions, subsequently creating a lipid film on the skin. In the given monitored intervals, observation was made of rapid onset of a hydration effect for both base emulsions, in comparison with the emulsions supplemented with vegetable oils. These emulsions with oils were slower to increase the amount of water in the stratum corneum, which was actually due to creation of a greasier film on the surface of the skin. Such a tendency for rise in hydration is very similar in the $\mathrm{o} / \mathrm{w}$ and $\mathrm{w} / \mathrm{o}$ emulsions. It was found that solely the base emulsions exhibited very good efficiency. Incorporating vegetable oils in these bases did not really contribute to their potential for hydration. It may be stated that the formulations supplemented with vegetable oils improved the emollient properties of the skin. The type of vegetable oil used did not appear to effect variation in the degree of hydration either, probably as a consequence of its low concentration in the emulsion systems, as stated in a paper (Smaoui et al., 2012). After degreasing and applying the studied formulations, the skin underwent gradual regeneration up to values corresponding with normally hydrated skin, $>45$ a. u., (Courage and Khazaka, 2010). During the measurement, the ability for absorption by the emulsion formulations containing vegetable oils was also monitored. Those most rapidly absorbed by the skin were emulsions supplemented with rosehip (Rosa rubiginosa), almond (Prunus amygdalus dulcis) and hemp (Cannabis sativa) oils. Unsaturated fatty acids of vegetable oils easily penetrate the intercellular lipid lamellae of the skin, replacing endogenous fatty acids and thus enhancing hydration of the horny layer of the skin and its barrier. Lodén and Andersson (1996) reached a similar conclusion by studying the impact of lipids on SLS-irritated skin. Arsić et al. (2012), during an in vivo double-blind randomized study, investigated the effectiveness of three o/w creams containing St. John's Wort oil extract as an active ingredient in olive, palm and sunflower oils, through the use of a sodium lauryl sulphate test. Their results clearly indicated the impact of the vegetable oils utilized. At the same time, they pointed out the influence of the type of oil used, especially of its chemical composition, i.e. composition of fatty acids, on the level of hydration of degreased skin.

The effectiveness of the barrier properties of the emulsion systems is clear in Figures $2 \mathrm{a}$ and $\mathrm{b}$. During the monitored intervals, the amount of water evaporated from the skin slightly decreased in the case of both basic formulations and formulations with vegetable oils. The least ability to decrease water evaporation from the epidermis was observed in the emulsion with sunflower (Hellianthus annuus) oil. In particular, it is oils with a high proportion of linoleic acid that play an important part in preserving epidermal integrity, thanks to the cohesion of the stratum corneum and prevention of TEWL (Berbis, Hesse, Privat, 1990). It was observed that after applying emulsions to the skin, a greater percentage of water evaporation (the evaporation phase) occurs as a result of the lipidization phase, during which lipids from the emulsion penetrate the epidermis, thereby boosting hydration of the skin (Arsić et al., 2012). Additionally, this finding correlates with the abilities to hydrate and concurrently retain water in the epidermis, as observed for the hydrophilic and lipophilic formulations utilized herein. Skin treated with said lipophilic formulations demonstrated reduced water loss from the epidermis. It is also more obvious than in the hydrophilic emulsions how adding vegetable oils enhanced the barrier properties of the lipophilic emulsions. Indeed, the barrier effect ended up being comparable to the monitored duration of the effect of the emulsions. It could be speculated that, in the lipidization phase, unsaturated fatty acids from the oil phase penetrate the intercellular lipid lamellae, owing to their good mobility, far more easily than saturated fatty acids (Lodén, Andersson, 1996; Prottey 1976; Prottey et 
al., 1975) are incorporated in the horny layer; in addition, such unsaturated fatty acids support or replace the endogenous fatty acids in the intercellular bilayers, thus repairing the skin barrier and subsequently increasing hydration of the skin (Denda et al., 1994).

Both of the tested emulsions exhibited a positive impact on the acidity of the skin. The slightly acidic to neutral $\mathrm{pH} 4.0-5.5$ indicates that the skin is in good condition (Ali, Yosipovitch, 2013). The cosmetic emulsions applied herein supplemented with vegetable oils actually altered the acidic $\mathrm{pH}$ of the skin in the majority of volunteers, veering towards neutrality (see Figure 3).

\section{CONCLUSIONS}

The findings presented in this study show that hydrophilic and lipophilic emulsion with vegetable oils exhibit good stability and biophysical characteristics. The

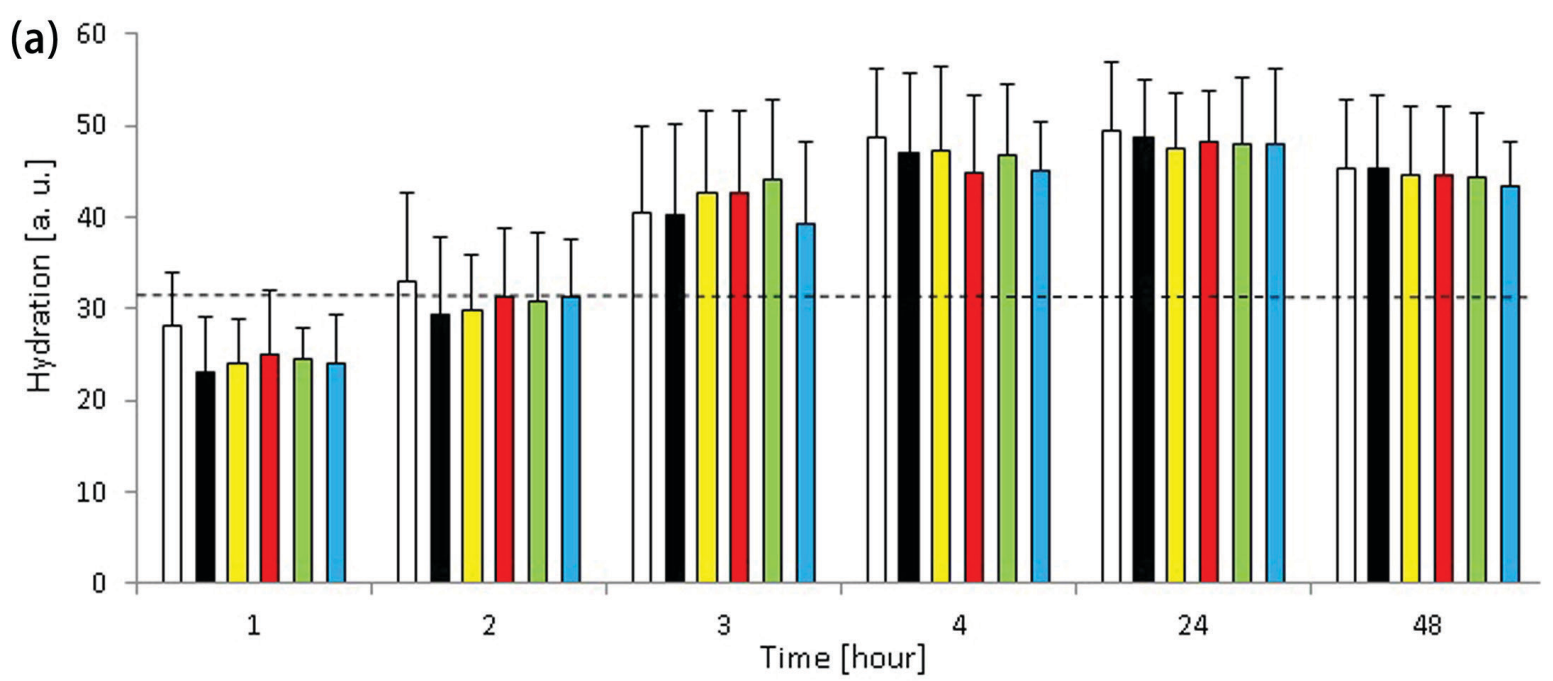
$\square 0 / w$ Emulsion base (EB)
D o/w EB+Meadowfoam Oil
$\square$ o/w EB+Prunus Am ygdalus Dulcis Oil
$\square \mathrm{o} / \mathrm{w} \mathrm{EB}+\mathrm{Cannabis}$ Sativa Oil
$\square \mathrm{o} / \mathrm{w}$ EB+Rosa Rubiginosa Oil
$\square$ o/w EB+Helianthus Annuus Oil

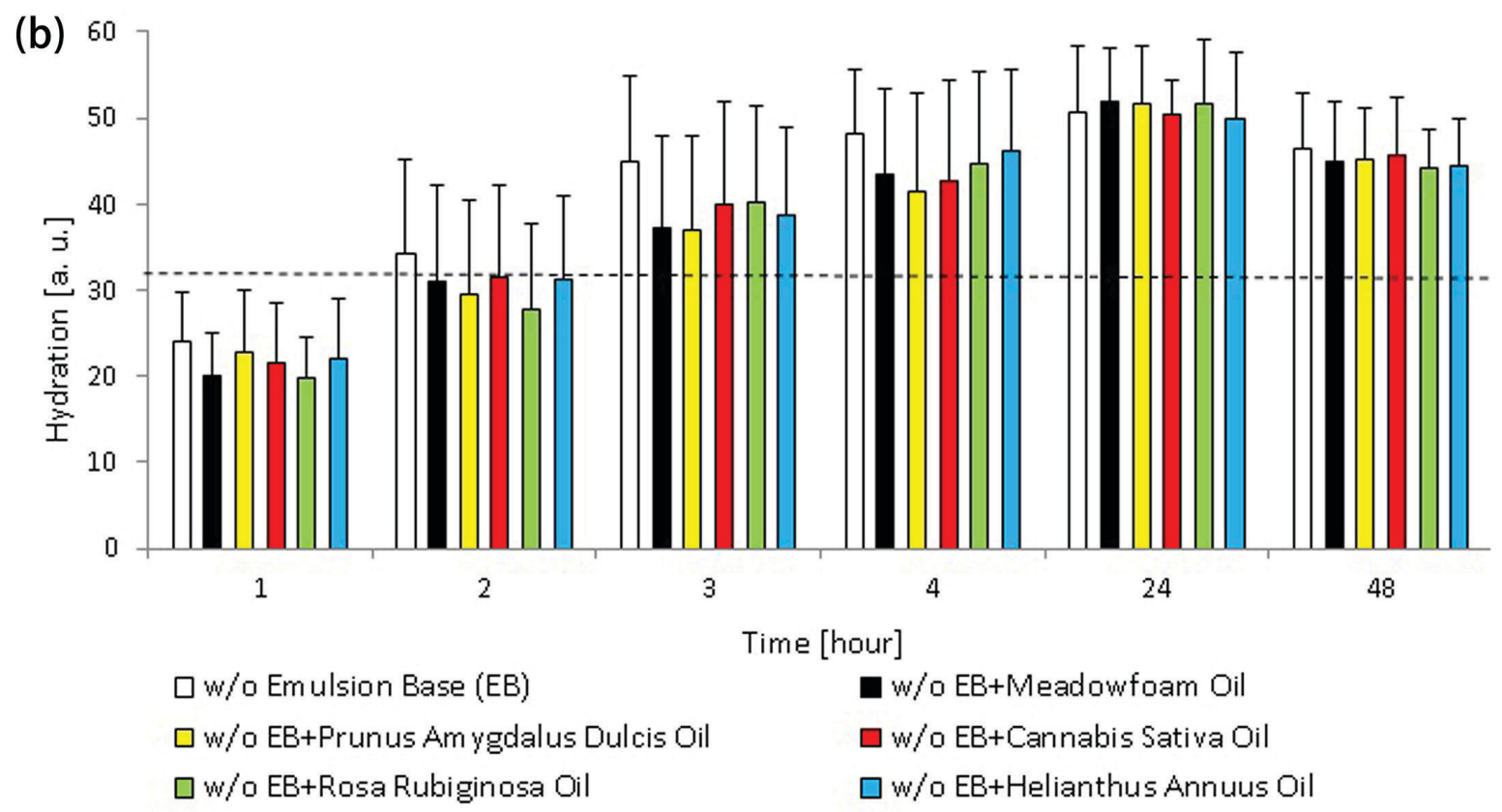

FIGURE 1 - Hydration effect of tested emulsions after SLS pre-treatment over the studied period, (a) o/w emulsions, (b) w/o emulsions. 

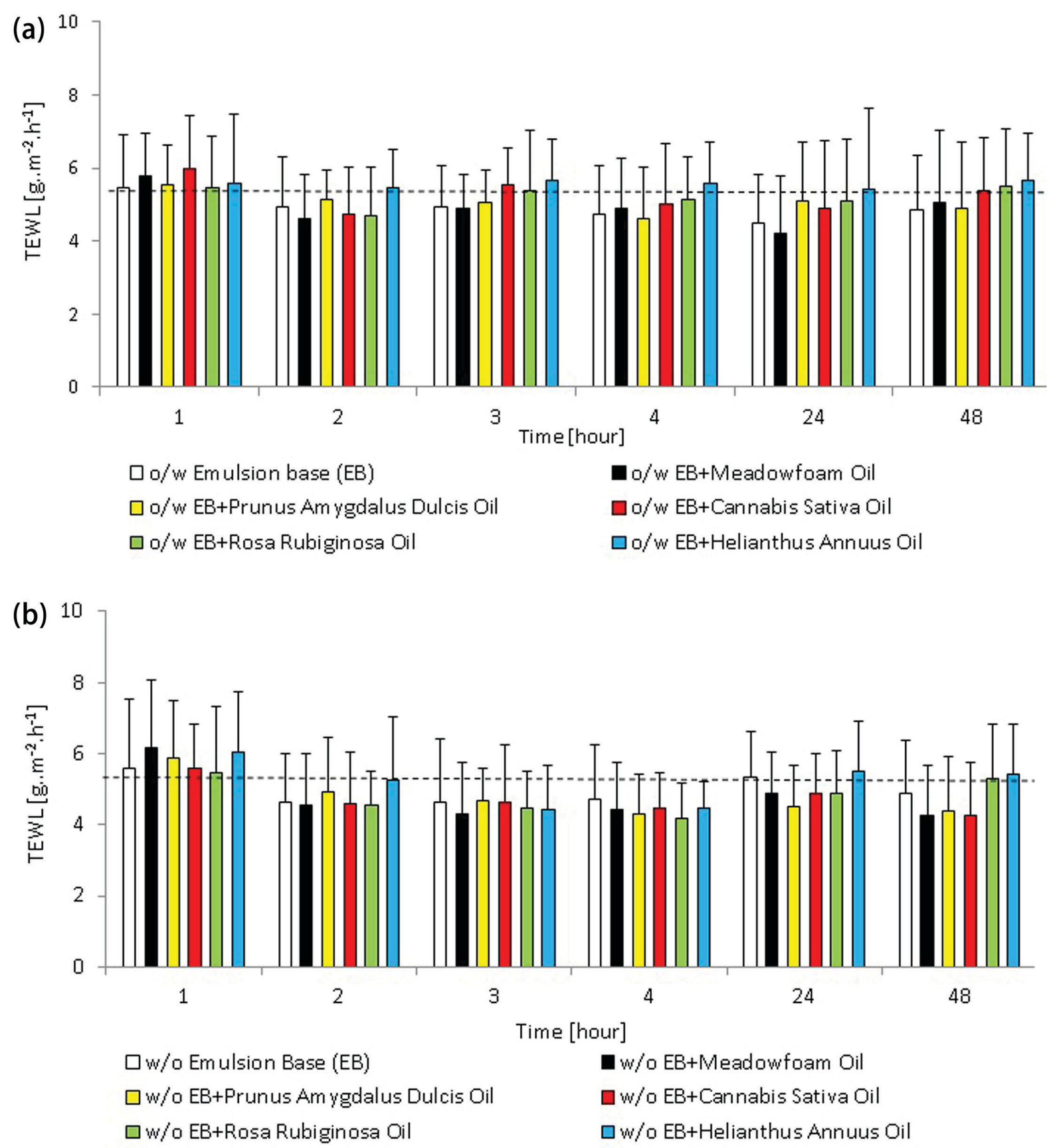

FIGURE 2 - TEWL after SLS pre-treatment and application of the tested emulsions over the studied period, (a) o/w emulsions, (b) w/o emulsions.

emulsion formulations with the addition of vegetable oils and the pure base itself were subjected to various storage conditions. The base emulsion and most of the tested formulations containing vegetable oils did not destabilize at storage temperatures of up to $37^{\circ} \mathrm{C}$. Only emulsions containing hemp (Cannabis sativa) and rosehip (Rosa rubiginosa) oils require that the storage temperature should remain below $25^{\circ} \mathrm{C}$. Consequently, the stability of these formulations could be supported by a more suitable emulsifier. Evaluating the biophysical properties of the studied emulsion systems shows that hydrophilic emulsions were absorbed by the skin faster than lipophilic emulsions, which means they also possessed superior moisturising effectiveness. The emulsion bases themselves also displayed very good hydrating properties. Studied vegetable oils as a form of additive did not significantly 

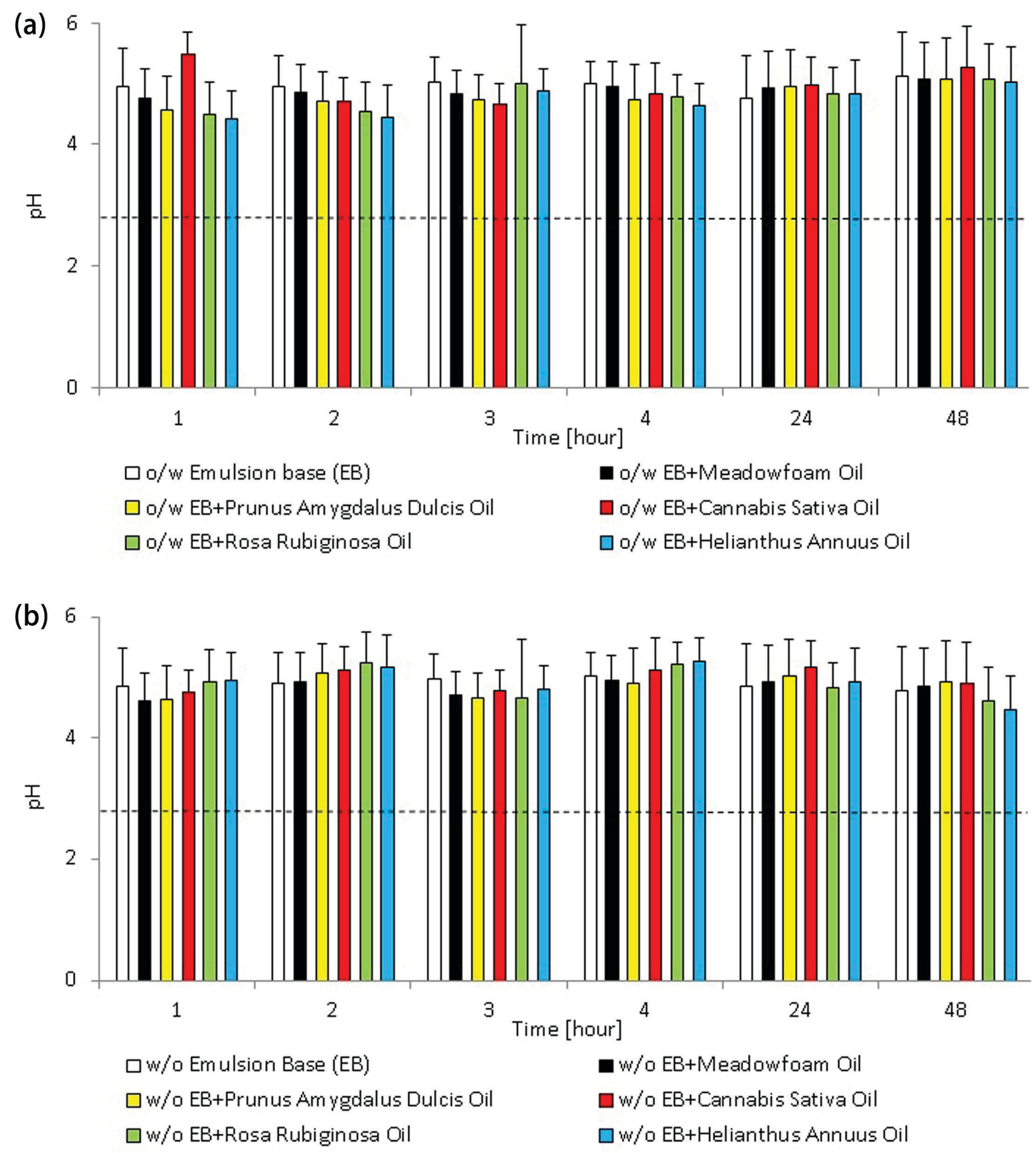

FIGURE 3 - Values for $\mathrm{pH}$ after SLS pre-treatment and application of the tested emulsions over the studied period, (a) o/w emulsions, (b) w/o emulsions.

enhance the moisturising activity of the emulsion bases. As for the concentration of the vegetable oils incorporated in the emulsion systems, the differences in hydration between the various formulations were minimal. Lipophilic emulsions were more effective at retaining epidermal water, as they created a lipid film on the surface of the skin. All tested formulations regulated skin $\mathrm{pH}$ towards neutrality.

\section{REFERENCES}

Alander J, Andersson A-CH, Lindström CH. Cosmetic emollients with high stability against photo-oxidation. Lipid Technol. 2006;18(10):226-30.

Ali SM, Yosipovitch G. Skin pH: From basic science to basic skin care. Acta Derm Venereol. 2013;93(3):261-67. 
Alvarez AMR, Rodriguez MLG. Lipids in pharmaceutical and cosmetic preparation. Grasas y Aceites. 2000;51(1-2):74-96.

Arsić I, Žugić A, Tadić V, Tasić-Kostov M, Mišić D, Primorac M, Runjaić-Antić D. Estimation of dermatological application of creams with St. John's Wort oil extracts. Molecules. 2012;17(1):275-94.

Benatkova N. Treatment of atopic dermatitis/eczema in adults. Med Pro Praxi. 2010;7(5):221-28.

Courage and Khazaka electronic GmbH. Information and operating instructions for the multi probe adapter MPA and its probes. Germany: Köln; 2010.

Berbis P, Hesse S, Privat Y. Essential fatty acids and the skin. Allerg Immunol. 1990;22(6):225-31.

Deferne JL, Pate DW. Hemp seed oil: A source of valuable essential fatty acids. J Int Hemp Assoc. 1996;3(1):4-7.

De Paepe K, Derde M-P, Roseeuw D, Rogiers V. Claim substantion and efficiency of hydrating body lotions and protective creams. Contact Dermatitis. 2000;42(4):227-34.

Denda M, Koyama A, Namba R, Horii I. Stratum corneum lipid morphology and transepidermal water loss in normal skin and surfactant-induced scaly skin. Arch Dermatol Res. 1994;286(1):41-46.

Draelos ZD. Cosmetic dermatology: Products and procedures. West Sussex: Wiley-Blackwell; 2010. 548 p.

Fertekova V. Cosmetics in theory and practice. Prague: Maxdorf; 2005. $341 \mathrm{p}$.

Ilyasoglu, H. Characterization of rosehip (Rosa canina L.) seed and seed oil. Int J Food Prop. 2014;17(7):1591-98.

Imhof A, Pine DJ. Stability of nonaqueous emulsions. J Colloid Interf Sci. 1997;192(2):368-74.

International ethical guidelines for health-related research involving humans. Geneva: Council for International Organizations of Medical Sciences (CIOMS); 2016.

Khan BA, Akhtar N, Mahmood T, Qayum M, Zaman SU. Formulation and pharmaceutical evaluation of a W/O emulsion of hippophae ramnoides fruit extract. J Pharm Res. 2010;3(6):1342-44.
Kusmirek J. Liquid sunshine: vegetable oils for aromatherapy. Glastonbury: Floramicus; 2002. 192 p.

Leizer C, Ribnicky D, Dushenkov S, Raskin I. The composition of hemp seed oil and its potential as an important source of nutrition. J Nutraceut Funct Med Foods. 2000;2(4):35-53.

Lévêque JL, De Rigal J, Saint-Léger D, Billy D. How does sodium lauryl sulfate alter the skin barrier function in man? A multiparametric approach. Skin Pharmacol. 1993;6(2):111-15.

Lodén M, Andersson AC. Effect of topically applied lipids on surfactant-irritated skin. Br J Dermatol. 1996;134(2):215-20.

Lodén M, Wessman C. The influence of a cream containing 20\% glycerin and its vehicle on skin barrier properties. Int J Cosmet Sci. 2001;3(2):115-19.

Masmoudi H, Dréau Le Y, Piccerelle P, Kister J. The evaluation of cosmetic and pharmaceutical emulsions aging process using classical techniques and a new method: FTIR. Int J Pharm. 2005;289(1-2):117-31.

Miller L, Miller B. Ayurveda \& aromatherapy: The earth essential guide to ancient wisdom and modern healing. Delhi: Motilal Banarsidass; 2017. 368 p.

Miraliakbari H, Shahidi F. Lipid class compositions, tocopherols and sterols of tree nut oils extracted with different solvents. J Food Lipids. 2008;15(1):81-96.

Miwa TK, Wolff IA. Fatty acids, Fatty alcohols, and wax esters from Limnanthes douglasii (Meadowfoam) seed oil. J Am Oil Chem Soc. 1962;39(7):320-22.

Orsavova J, Misurcova L, Vavra-Ambrozova J, Vicha R, Mlcek $\mathrm{J}$. Fatty acids composition of vegetable oils and its contribution to dietary energy intake and dependence of cardiovascular mortality on dietary intake of fatty acids. Int J Mol Sci. 2015;16(6):12871-90.

Polaskova J, Pavlackova J, Egner P. Effect of vehicle on the performance of active moisturizing substances. Skin Res Tech. 2015;21(4):403-412.

Prescha A, Grajzer M, Dedyk M, Grajeta H. The antioxidant activity and oxidative stability of cold-pressed oils. J Am Oil Chem Soc. 2014;91:1291-301.

Prottey C. Essential fatty acids and the skin. Br J Dermatol. 1976;94(5):579-87. 
Prottey C, Hartop PJ, Press M. Correction of the cutaneous manifestations of essential fatty acid deficiency in man by application of sunflower-seed oil to the skin. J Invest Dermatol. 1975;64(4):228-34.

Rafalowski R, Zegarska Z, Kuncewicz A, Borejszo Z. Fatty acid composition, tocopherols and $\beta$-carotene content in Polish commercial vegetable oils. Pak J Nutr. 2008;7(2):278-82.

Rele AS, Mohile RB. Effect of mineral oil, sunflower oil, and coconut oil on prevention of hair damage. J Cosmet Sci. 2003;54(2):175-92.

Smaoui S, Hlima BH, Jarraya R, Kamoun NG, Elouze R, Damak M. Cosmetic emulsion from virgin olive oil: Formulation and bio-physical evaluation. Afr J Biotechnol. 2012;11(40):9664-71.
Stamatas GN, Sterke J, Hauser M, von Stetten O, Pol van der A. Lipid uptake and skin occlusion following topical application of oils on adult and infant skin. J Dermatol Sci. 2008;50(2):135-42.

Zielińska A, Nowak I. Fatty acids in vegetable oils and their importance in cosmetic industry. Chemik. 2014;68(2):103-10.

Wolhman A. Meadowfoam seed oil derivative and its activity on human hair. Cosm Toiletries. 1997;112(8):83-88.

Received for publication on $30^{\text {th }}$ October 2017 Accepted for publication on $06^{\text {th }}$ February 2018 